\title{
On the Brézis-Nirenberg Problem
}

\author{
M. Schechter \& Wenming Zou
}

\author{
Communicated by C. DAFERMos
}

\begin{abstract}
We study the following Brézis-Nirenberg problem (Comm Pure Appl Math 36:437-477, 1983):

$$
-\Delta u=\lambda u+|u|^{2^{*}-2} u, \quad u \in H_{0}^{1}(\Omega),
$$

where $\Omega$ is a bounded smooth domain of $\mathbf{R}^{N}(N \geqq 7)$ and $2^{*}$ is the critical Sobolev exponent. We show that, for each fixed $\lambda>0$, this problem has infinitely many sign-changing solutions. In particular, if $\lambda \geqq \lambda_{1}$, the Brézis-Nirenberg problem has and only has infinitely many sign-changing solutions except zero. The main tool is the estimates of Morse indices of nodal solutions.
\end{abstract}

\section{Introduction}

We study the following Brézis-Nirenberg problem

$$
-\Delta u=\lambda u+|u|^{2^{*}-2} u, \quad u \in H_{0}^{1}(\Omega),
$$

where $\Omega$ is a bounded smooth domain of $\mathbf{R}^{N}(N \geqq 3), 2^{*}=\frac{2 N}{N-2}$ is the critical Sobolev exponent and $\lambda>0$. The pioneering paper on equation (1) was by BrézisNirenberg [7] in 1983 where the authors showed that for $N \geqq 4$ and $\lambda \in\left(0, \lambda_{1}\right)$ problem (1) has at least one positive solution. In the sequel, $\lambda_{1}$ denotes the principal eigenvalue of $-\Delta$ on $\Omega$. The same conclusion was proved in [7] for $N=3$ when $\Omega$ is a ball and $\lambda \in\left(\lambda_{1} / 4, \lambda_{1}\right)$. In this case, by using a version of the Pohozaev Identity, equation (1) has no radial solution when $\lambda \in\left(0, \lambda_{1} / 4\right)$. It is still an open question whether there exist sign-changing non-radial solutions to (1). Note that, by using the Pohozaev Identity, (1) has no nontrivial solution when $\lambda \leqq 0$ and $\Omega$ is star-shaped. Since 1983, there has been a considerable number of papers on problem (1). Let us now briefly recap the main results obtained to date. The first 
multiplicity result was obtained by Cerami et al. [8], in which they proved that the number of solutions of (1) is bounded below by the number of eigenvalues of $(-\Delta, \Omega)$ lying in the open interval $\left(\lambda, \lambda+S|\Omega|^{-2 / N}\right)$, where $S$ is the best constant for the Sobolev embedding $D^{1,2}\left(\mathbf{R}^{N}\right) \hookrightarrow L^{2^{*}}\left(\mathbf{R}^{N}\right)$ and $|\Omega|$ is the Lebesgue measure of $\Omega$. If $N \geqq 4$ and $\Omega$ is a ball, then for any $\lambda>0$, problem (1) has infinitely many sign-changing solutions which are built using particular symmetries of the domain $\Omega$ (see Fortunato and Jannelli [15]). If $N \geqq 7$ and $\Omega$ is a ball, then for each $\lambda>0$, problem (1) has infinitely many sign-changing radial solutions (see Solimini [23]). In the papers [15,23], the symmetry of the ball $=\Omega$ plays an essential role, therefore their methods are invalid for general domains. In Cerami et al. [9] it was proved for $N \geqq 6$, that (1) has two pairs of solutions on any smooth bounded domain. When $4 \leqq N \leqq 6$ and $\Omega$ is a ball, there is a $\lambda^{*}>0$ such that (1) has no radial solutions which change sign if $\lambda \in\left(0, \lambda^{*}\right)$ (see Atkinson et al. [1]). Very recently, Devillanova and Solimini [14] showed that, if $N \geqq 7$, problem (1) has infinitely many solutions for each $\lambda>0$. In [10], Clapp-Weth got only finitely many solutions to (1) for each $\lambda>0$ and $N \geqq 4$. Neither [14] nor [10] have information on the sign-changingness of the solutions thus obtained.

A natural question which seems to still be open is whether (1) has infinitely many sign-changing solutions on each bounded domain $\Omega$ and for any $\lambda>0$. This is expected by many experts but no proof has yet been obtained. The results of Atkinson et al. [1] suggest that the hypothesis $N>6$ should be imposed since it is needed in the radial case. In this paper, we give a positive answer to this open question. Precisely, we shall prove the following theorem.

\section{Theorem 1. If $N \geqq 7$, problem (1) has infinitely many sign-changing solutions.}

Remark 1. Note that if $\lambda \geqq \lambda_{1}$, any nontrivial solution of (1) is sign-changing. This can be seen by multiplying the first eigenfunction of the Dirichlet zero-boundary value problem and integrating both sides. Thus, by the results of [14], problem (1) has and only has infinitely many sign-changing solutions for this case.

To prove Theorem 1 , it suffices to consider the case of $\lambda \in\left(0, \lambda_{1}\right)$. To this goal, we first establish an abstract theorem on the estimate of the Morse index for signchanging critical points. We will introduce a new kind of linking below a level set which provides a sign-changing critical point via a minimax formula and then find the lower bound of its Morse index. Similar to the classical case, the lower bound of the Morse index is determined by the dimension of the subspace which may be chosen as large as we like. By combining this with the uniform bound theorem due to Devillanova and Solimini [14], we show that, for each fixed $\lambda>0$, (1) indeed has infinitely many sign-changing solutions.

The study of sign-changing solutions to some elliptic equations has been an increasing interest in recent years (cf. [2-6,12,13,17,21,22,26] and the references cited therein). As when finding an existence result in a classical case, the information on Morse index of sign-changing solutions can yield new conclusions. In paper [3] by using critical group and algebraic topology arguments, two kinds of Morse indices of sign-changing solutions were obtained: one is a sign-changing solution of the mountain pass type with Morse index less than or equal to 1; the other may 
be degenerate and has Morse index 2. In [2], an upper-bound for the Morse index of the sign-changing solution was obtained. The results of [2] mainly rely on singular homology groups. In [6], a least energy sign-changing solution with Morse index 2 is obtained. If the nonlinear term is odd, paper [6] also obtains a sequence $\left\{u_{k}\right\}$ of sign-changing solutions such that each $u_{k}$ has at most $k$ nodal domains (see also [5]). But, under those assumptions, the lower bound of the Morse index for each $u_{k}$ has not been determined. For many cases, the Morse indices of sign-changing critical points produced by general minimax procedure are not clear. Our goal here is to establish an estimate on the Morse index of sign-changing critical points.

\section{Morse index of sign-changing critical points}

Let $E$ be a Hilbert space with the inner product $\langle\cdot, \cdot\rangle$ and the corresponding norm $\|\cdot\|$. We assume that

$\left(\mathbf{A}_{\mathbf{0}}\right)$ there is another norm $\|\cdot\|_{\star}$ of $E$ such that $\|u\|_{\star} \leqq C_{0}\|u\|$ for all $u \in E$, where $C_{0}>0$ is a constant. Moreover, we assume that $\left\|u_{n}-u^{*}\right\|_{\star} \rightarrow 0$ whenever $u_{n} \rightarrow u^{*}$ weakly in $(E,\|\cdot\|)$.

Let $E:=\overline{\cup_{k=1}^{\infty} E_{k}}$ with $\operatorname{dim} E_{k}=k, E_{k} \subset E_{k+1}$. Let $G \in \mathbf{C}^{2}((E,\|\cdot\|), \mathbf{R})$ be an even functional which maps bounded sets to bounded sets in terms of the norm $\|\cdot\|$. In the sequel, all properties are with respect to the norm $\|\cdot\|$ if without specific indication. Assume that $G^{\prime \prime}\left(u_{0}\right)$ is Fredholm for any critical point $u_{0}$ of $G$. The gradient $G^{\prime}$ is of the form $G^{\prime}(u)=u-K_{G}(u)$, where $K_{G}: E \rightarrow E$ is a continuous operator. Let $\mathcal{K}:=\left\{u \in E: G^{\prime}(u)=0\right\}$ and $\tilde{E}:=E \backslash \mathcal{K}, \mathcal{K}[a, b]:=\{u \in \mathcal{K}: G(u) \in[a, b]\}$. Let $\mathcal{P}$ be a closed convex and weakly closed positive cone of $E$. We call the elements outside $\pm \mathcal{P}$ sign-changing. Assume that $\left( \pm \mathcal{P} \cap\left(E_{k}^{\perp} \backslash\{0\}\right)=\emptyset\right.$ for all $k \geqq 2$, that is, any nonzero element of $E_{k}^{\perp}$ is sign-changing. For each $\mu>0$, define $\mathcal{D}(\mu):=$ $\{u \in E: \operatorname{dist}(u, \mathcal{P})<\mu\}$. Then $\mathcal{D}(\mu)$ is an open convex set containing the positive cone $\mathcal{P}$ in its interior. Set $\mathcal{D}^{*}=\mathcal{D}^{*}(\mu):=\mathcal{D}(\mu) \cup(-\mathcal{D}(\mu)), \quad \mathcal{S}^{*}=E \backslash \mathcal{D}^{*}(\mu)$. We use the following assumptions.

(A) For any $\mu_{0}>0$ small enough, we have that $K_{G}\left( \pm \mathcal{D}\left(\mu_{0}\right)\right) \subset \pm \mathcal{D}(\mu) \subset$ $\pm \mathcal{D}\left(\mu_{0}\right)$ for some $\mu \in\left(0, \mu_{0}\right)$. Moreover, $\pm \mathcal{D}\left(\mu_{0}\right) \cap \mathcal{K} \subset \pm \mathcal{P}$.

(A2) For each $k, \lim _{\|u\| \rightarrow \infty, u \in E_{k}} G(u)=-\infty$.

(A) Assume that for any $\alpha_{1}, \alpha_{2}>0$ there exists an $\alpha_{3}$ depending on $\alpha_{1}$ and $\alpha_{2}$ such that $\|u\| \leqq \alpha_{3}$ for all $u \in G^{\alpha_{1}} \cap\left\{u \in E:\|u\|_{\star} \leqq \alpha_{2}\right\}$, where $G^{\alpha_{1}}=\left\{u \in E: G(u) \leqq \alpha_{1}\right\}$.

Conditions similar to $\left(A_{1}\right)$ have been used in $[5,6,12,13,17,21,22,26]$. Let

$$
C_{k+1}^{* *}:=\sup _{E_{k+1}} G \text {. }
$$

Then by condition $\left(A_{2}\right), C_{k+1}^{* *}$ is well-defined and $C_{k+1}^{* *}<\infty$. Write $E=E_{k} \oplus E_{k}^{\perp}$ and let

$$
\beta^{*}(u)=\left\{\begin{array}{cc}
\frac{\|u\|\|u\|_{\star}}{\|u\|+\|u\|_{\star}}, & u \neq 0 \\
0, & u=0
\end{array}\right.
$$


then $\beta^{*}: E \rightarrow E$ is continuous. Set $S_{0}(k):=\left\{u \in E_{k}^{\perp}: \beta^{*}(u)=1\right\}$, then it is easy to check that there is a constant $\alpha_{4}>0$ such that $\|u\|_{\star} \leqq \alpha_{4}$ for all $u \in S_{0}(k)$. Set $S(k):=S_{0}(k) \cap G^{C_{k+1}^{* *}}$.

Lemma 1. By assumption $\left(A_{3}\right)$, we have a constant $\alpha_{5}=\alpha_{5}\left(\alpha_{4}, C_{k+1}^{* *}\right)>0$ such that $\|u\| \leqq \alpha_{5}$ for all $u \in S(k)$. Hence, there is an $\Lambda_{0}=\Lambda_{0}\left(\alpha_{5}\right)>0$ such that $\inf _{u \in S(k)} G \geqq-\Lambda_{0}$.

Lemma 2. There is a constant $\delta>0$ such that $\operatorname{dist}(S(k), \pm \mathcal{P})=\delta>0$.

Proof. We begin with ideas initiated in [21]. By negation, we assume that

$$
\operatorname{dist}(S(k), \mathcal{P})=0 \text {. }
$$

Then we find $\left\{u_{n}\right\} \subset S(k),\left\{p_{n}\right\} \subset \mathcal{P}$ such that $\left\|u_{n}-p_{n}\right\| \rightarrow 0$. Then $\left\{u_{n}\right\}$, hence $\left\{p_{n}\right\}$, is bounded in both $(E,\|\cdot\|)$ and $\left(E,\|\cdot\|_{\star}\right)$. We assume that $u_{n} \rightarrow u^{*} \in$ $E, \quad p_{n} \rightarrow p^{*} \in \mathcal{P}$ weakly in $(E,\|\cdot\|) ; u_{n} \rightarrow u^{*}$ strongly in $\left(E,\|\cdot\|_{\star}\right)$. Then we observe that $u^{*} \in E_{k}^{\perp}$. Since $\frac{\left\|u_{n}\right\|\left\|u_{n}\right\|_{\star}}{\left\|u_{n}\right\|+\left\|u_{n}\right\|_{\star}}=1$ and $\left\|u_{n}-u^{*}\right\|_{\star} \rightarrow 0$, we see that $u^{*} \neq 0$. However, since $u^{*}=p^{*}$, we get a contradiction, since all nonzero elements of $E_{k}^{\perp}$ are sign-changing.

Note that because both $\|\cdot\|_{\star}$ and $\|\cdot\|$ are equivalent in $E_{k+1}$, we have a constant $\varrho_{k+1}$ such that $\|u\| \leqq \varrho_{k+1}\|u\|_{\star}$ for all $u \in E_{k+1}$. Let

$$
\Gamma_{k+1}^{*}=\left\{h: \quad h \in \mathbf{C}\left(\Theta_{k+1}, E\right),\left.h\right|_{\partial \Theta_{k+1}}=\mathbf{i d}, h \text { is odd }\right\},
$$

where $\Theta_{k+1}:=\left\{u \in E_{k+1}:\|u\| \leqq R_{k+1}\right\}$. Further, by Lemma 1 and condition $\left(A_{2}\right)$, we may choose $R_{k+1}$ large enough such that

$$
\partial \Theta_{k+1} \cap S(k)=\emptyset, \quad \sup _{\partial \Theta_{k+1}} G<<-\Lambda_{0} \leqq \inf _{S(k)} G, \quad R_{k+1} \geqq \varrho_{k+1}+2 .
$$

Without loss of generality, we may assume that $R_{k+2}>R_{k+1}$.

Lemma 3. $h\left(\Theta_{k+1}\right) \cap S_{0}(k) \neq \emptyset, \quad \forall h \in \Gamma_{k+1}^{*}$.

Proof. Let $U:=\left\{u \in E_{k+1}: \beta^{*}(h(u))<1\right\} \cap\left\{u \in E_{k+1}:\|u\|<R_{k+1}\right\}$, then $U$ is a symmetric neighborhood of zero in $E_{k+1}$. Let $P: E \rightarrow E_{k}$ be the orthogonal projection; then $P \circ h: \partial U \rightarrow E_{k}$ is odd and continuous. By BorsukUlam theorem, we have that $P \circ h(u)=0$ for some $u \in \partial U$. Hence, $h(u) \in E_{k}^{\perp}$. We claim $u \notin \partial\left\{u \in E_{k+1}:\|u\|<R_{k+1}\right\}$. Otherwise, $\|u\|=R_{k+1}$ and then $h(u)=u, P(u)=0$. But $\frac{\|h(u)\|\|h(u)\|_{\star}}{\|h(u)\|+\|h(u)\|_{\star}} \leqq 1$, it follows that $R_{k+1}=\|u\| \leqq$ $1+\frac{\|u\|}{\|u\|_{\star}} \leqq 1+\varrho_{k+1}$. This is impossible, so our claim is true. This means that $u \in \partial\left\{u \in E_{k+1}: \beta^{*}(h(u))<1\right\},\|u\| \leqq R_{k+1}, u \in E_{k+1}$. Hence, $h(u) \in$ $E_{k}^{\perp}, \frac{\|h(u)\|\|h(u)\|_{\star}}{\|h(u)\|+\|h(u)\|_{\star}}=1$, that is, $h(u) \in S_{0}(k)$.

Combining the definition of $\mathcal{S}^{*}$ and Lemma 2, we may choose $\mu_{0}$ in $\left(A_{1}\right)$ such that $S(k) \subset \mathcal{S}^{*}=E \backslash \mathcal{D}^{*}\left(\mu_{0}\right)$. 
Definition 1. A compact symmetric subset $A$ of $E$ with $\partial \Theta_{k+1} \subset A$ is said to be linked to $S_{0}(k)$ if, for any continuous mapping $h \in \mathbf{C}([0,1] \times A, E)$ satisfying

- $h(t, u)$ is odd in $u \in A$,

- $h(t, u)=u$ for all $u \in \partial \Theta_{k+1}$,

there holds $h(1, A) \cap S_{0}(k) \neq \emptyset$.

Define

$$
\mathcal{L}:=\left\{A \subset G^{C_{k+1}^{* *}}: \quad A \quad \text { is linked to } \quad S_{0}(k)\right\} .
$$

Then $\mathcal{L} \neq \emptyset$, since by Lemma 3 and the definition of $S_{0}(k)$ we see that $\Theta_{k+1} \in \mathcal{L}$. Note that $h(t, \cdot): \equiv \mathbf{i d}$ is a mapping satisfying the properties of Definition 1 .

Theorem 2. Suppose that $G$ satisfies the (PS) condition and assumptions $\left(A_{1}\right)-\left(A_{3}\right)$. Define

$$
C^{*}=\inf _{A \in \mathcal{L}} \sup _{A \cap \mathcal{S}^{*}} G(u) .
$$

Then $C^{*} \in\left[-\Lambda_{0}, C_{k+1}^{* *}\right]$ and $G$ has a sign-changing critical point $u^{*} \in \mathcal{S}^{*}\left(u^{*} \neq 0\right)$ at level $C^{*}$ and the augmented Morse index $m^{*}\left(u^{*}\right)$ of $u^{*}$ is greater than or equal to $k$.

Proof. Since $A$ links $S_{0}(k)$, by Definition 1 , we observe that $A \cap S_{0}(k) \neq \emptyset$. Note that $A \subset G^{C_{k+1}^{* *}}$, hence $A \cap S(k) \neq \emptyset$ and then $C^{*}$ is well defined. Then for any $A \in \mathcal{L}$, by (2), $\sup _{A \cap \mathcal{S}^{*}} G \geqq \inf _{S(k)} G \geqq-\Lambda_{0}>>\sup _{u \in \partial \Theta_{k+1}} G$. Therefore, $C^{*} \geqq-\Lambda_{0}$. Evidently, we have $C^{*} \leqq \sup G(A) \leqq C_{k+1}^{* *}$. We divide the proof into steps.

Step 1. We show that there exists a sign-changing critical point $0 \neq u^{*} \in \mathcal{S}^{*}$ with $G\left(u^{*}\right)=C^{*}$. We prove that $\mathcal{K}\left[C^{*}-\bar{\varepsilon}, C^{*}+\bar{\varepsilon}\right] \cap \mathcal{S}^{*} \neq \emptyset$ for all $\bar{\varepsilon}>0$ small enough. That is, there is a sign-changing critical point at level $C^{*}$. By negation, we assume that $\mathcal{K}\left[C^{*}-\bar{\varepsilon}, C^{*}+\bar{\varepsilon}\right] \cap \mathcal{S}^{*}=\emptyset$ for some $\bar{\varepsilon}>0$, then by $\left(A_{1}\right), \mathcal{K}\left[C^{*}-\bar{\varepsilon}, C^{*}+\bar{\varepsilon}\right] \subset$ $(-\mathcal{P} \cup \mathcal{P})$. We first assume $\mathcal{K}\left[C^{*}-\bar{\varepsilon}, C^{*}+\bar{\varepsilon}\right] \neq \emptyset$. Since $\mathcal{K}\left[C^{*}-\bar{\varepsilon}, C^{*}+\bar{\varepsilon}\right]$ is compact in $E$, by the definition of $\mathcal{S}^{*}$, we must have $\operatorname{dist}\left(\mathcal{K}\left[C^{*}-\bar{\varepsilon}, C^{*}+\bar{\varepsilon}\right], \mathcal{S}^{*}\right):=$ $\delta_{0}>0$. By the (PS) condition, there is an $\varepsilon_{1} \in(0, \bar{\varepsilon} / 3), \varepsilon_{1}<1$ such that $\left\|G^{\prime}(u)\right\| \geqq$ $\varepsilon_{1}$ for $u \in G^{-1}\left[C^{*}-\varepsilon_{1}, C^{*}+\varepsilon_{1}\right] \backslash\left(\mathcal{K}\left[C^{*}-\bar{\varepsilon}, C^{*}+\bar{\varepsilon}\right]\right)_{\delta_{0} / 2}$, here and in the sequel, $(A)_{c}:=\{u \in E: \operatorname{dist}(u, A) \leqq c\}$. By decreasing $\varepsilon_{1}$, we may assume that $3 \varepsilon_{1}<C^{*}-\sup _{\partial \Theta_{k+1}} G$. Then $\left\langle G^{\prime}(u), W(u)\right\rangle \geqq \varepsilon_{1}^{2} / 16$ for any $u$ with

$$
u \in G^{-1}\left[C^{*}-\varepsilon_{1}, C^{*}+\varepsilon_{1}\right] \backslash\left(\mathcal{K}\left[C^{*}-\bar{\varepsilon}, C^{*}+\bar{\varepsilon}\right]\right)_{\delta_{0} / 2},
$$

where $W(u)=\frac{(1+\|u\|)^{2} Y(u)}{(1+\|u\|)^{2}\|Y(u)\|^{2}+1}$ and $Y(u)=u-L_{0}(u)$ is the odd pseudo-gradient vector field of $G:\left\langle G^{\prime}(u), Y(u)\right\rangle \geqq \frac{1}{2}\left\|G^{\prime}(u)\right\|^{2}$ and $\|Y(u)\| \leqq 2\left\|G^{\prime}(u)\right\|$ for all $u \in \tilde{E}$, where $L_{0}\left( \pm \mathcal{D}_{\mu_{0}}\right) \subset \pm \mathcal{D}_{\mu_{0}}$ (By $\left(A_{1}\right)$, this can be done as that in [22]). Let

$$
U_{1}=\left\{u \in E:\left|G(u)-C^{*}\right| \geqq 3 \varepsilon_{1}\right\}, \quad U_{2}=\left\{u \in E:\left|G(u)-C^{*}\right| \leqq 2 \varepsilon_{1}\right\} .
$$


Let $y(u): E \rightarrow[0,1]$ be a locally Lipschitz continuous map such that

$$
y(u):= \begin{cases}1 & \text { for all } u \in E \backslash\left(\mathcal{K}\left[C^{*}-\bar{\varepsilon}, C^{*}+\bar{\varepsilon}\right]\right)_{\delta_{0} / 2} \\ 0 & \text { for all } u \in\left(\mathcal{K}\left[C^{*}-\bar{\varepsilon}, C^{*}+\bar{\varepsilon}\right]\right)_{\delta_{0} / 3} .\end{cases}
$$

Consider $h(u):=\frac{\operatorname{dist}\left(u, U_{1}\right)}{\operatorname{dist}\left(u, U_{2}\right)+\operatorname{dist}\left(u, U_{1}\right)}$. Let $\bar{W}(u):=y(u) h(u) W(u)$ if $u \in \tilde{E}$ and $\bar{W}(u)=0$ otherwise, then $\bar{W}$ is a locally Lipschitz vector field on $E$. We consider the following Cauchy initial value problem: $\frac{\mathrm{d} \eta(t, u)}{\mathrm{d} t}=-\bar{W}(\eta(t, u))$ with $\eta(0, u)=u \in E$, which has a unique continuous odd solution $\eta(t, u)$ in $E$. Evidently, $G(\eta(t, u))$ is non-increasing in $t$. By the definition of $C^{*}$, there exists an $A \in \mathcal{L}$ such that $\sup _{A \cap \mathcal{S}^{*}} G \leqq C^{*}+\varepsilon_{1}$. Therefore, $A \subset G^{C^{*}+\varepsilon_{1}} \cup\left(E \backslash \mathcal{S}^{*}\right)$. We claim that there exists a $T_{1}>0$ such that $\eta\left(T_{1}, A\right) \subset G^{C^{*}-\varepsilon_{1} / 4} \cup \mathcal{D}^{*}$. In fact, if $u \in A \cap \mathcal{D}^{*}$, then similar to [22] (see also [10,12]), $\eta(t, u) \in \mathcal{D}^{*}$ for all $t \geqq 0$. If $u \in$ $A, u \notin \mathcal{D}^{*}$, then we see that $G(u) \leqq C^{*}+\varepsilon_{1}$. If $G(u) \leqq C^{*}-\varepsilon_{1}$, then $G(\eta(t, u)) \leqq$ $G(u) \leqq C^{*}-\varepsilon_{1}$ for all $t \geqq 0$. If $G(u)>C^{*}-\varepsilon_{1}$, then $u \in G^{-1}\left[C^{*}-\varepsilon_{1}, C^{*}+\varepsilon_{1}\right]$. If dist $\left(\eta([0, \infty), u), \mathcal{K}\left[C^{*}-\bar{\varepsilon}, C^{*}+\bar{\varepsilon}\right]\right) \leqq \delta_{0} / 2$, then there exists a $t_{m}$ such that $\eta\left(t_{m}, u\right) \notin \mathcal{S}^{*}$. Moreover, we may choose $m$ so that $\operatorname{dist}\left(\eta\left(t_{m}, u\right), \mathcal{S}^{*}\right) \geqq \frac{1}{3} \delta_{0}>0$. Assume dist $\left(\eta([0, \infty), u), \mathcal{K}\left[C^{*}-\bar{\varepsilon}, C^{*}+\bar{\varepsilon}\right]\right)>\delta_{0} / 2$. Similarly, we assume that $G(\eta(t, u))>C^{*}-\varepsilon_{1}$ for all $t$ (otherwise, we are done), then $\eta(t, u) \in G^{-1}\left[C^{*}-\right.$ $\left.\varepsilon_{1}, C^{*}+\varepsilon_{1}\right] \backslash\left(\mathcal{K}\left[C^{*}-\bar{\varepsilon}, C^{*}+\bar{\varepsilon}\right]\right)_{\delta_{0} / 2}$, hence, $h(\eta(t, u))=1, y(\eta(t, u))=1$ and $\left\langle G^{\prime}(\eta(t, u)), W(\eta(t, u))\right\rangle \geqq \varepsilon_{1}^{2} / 16$ for all $t \geqq 0$. Therefore, $G\left(\eta\left(\frac{48}{\varepsilon_{1}}, u\right)\right)=$ $G(u)+\int_{0}^{\frac{48}{\varepsilon_{1}}} \mathrm{~d} G(\eta(s, u)) \leqq C^{*}-2 \varepsilon_{1}$. By combining the above arguments, for any $u \in A \backslash \mathcal{D}^{*}$, there exists a $T_{u}>0$ such that either $\eta\left(T_{u}, u\right) \in G^{C^{*}-\varepsilon_{1} / 2}$ or $\operatorname{dist}\left(\eta\left(T_{u}, u\right), \mathcal{S}^{*}\right) \geqq \frac{1}{3} \delta_{0}$. By continuity, there exists a neighborhood $U_{u}$ of $u$ such that either $\eta\left(T_{u}, U_{u}\right) \subset G^{C^{*}-\varepsilon_{1} / 3}$ or $\operatorname{dist}\left(\eta\left(T_{u}, U_{u}\right), \mathcal{S}^{*}\right) \geqq \frac{1}{4} \delta_{0}$. Both cases imply that $\eta\left(T_{u}, U_{u}\right) \subset G^{C^{*}-\varepsilon_{1} / 3} \cup\left(E \backslash \mathcal{S}^{*}\right)$. Since $A \backslash \mathcal{D}^{*}$ is compact in $E$, we get a $T_{1}>0$ such that

$$
\eta\left(T_{1}, A\right) \subset G^{C^{*}-\varepsilon_{1} / 4} \cup\left(E \backslash \mathcal{S}^{*}\right)
$$

If $\mathcal{K}\left[C^{*}-\bar{\varepsilon}, C^{*}+\bar{\varepsilon}\right]=\emptyset$, then we choose $\left(\mathcal{K}\left[C^{*}-\bar{\varepsilon}, C^{*}+\bar{\varepsilon}\right]\right)_{\delta_{0} / 2}=\emptyset$ and trivially, (3) is still true. Now we show that $\eta\left(T_{1}, A\right) \in \mathcal{L}$. Actually, note $\partial \Theta_{k+1} \subset$ $A \in \mathcal{L}$ and $\partial \Theta_{k+1} \subset U_{1}$, then $\left.\eta(t, \cdot)\right|_{\partial \Theta_{k+1}}=$ id for all $t \geqq 0$. Then $\partial \Theta_{k+1} \subset$ $\eta\left(T_{1}, A\right)$. On the other hand, $\sup G\left(\eta\left(T_{1}, A\right)\right) \leqq \sup G(A) \leqq C_{k+1}^{* *}$. For any $h \in$ $\mathbf{C}\left([0,1] \times \eta\left(T_{1}, A\right), E\right)$ with $h(t, u)$ odd in $u \in \eta\left(T_{1}, A\right)$ and $\left.h(t, \cdot)\right|_{\partial \Theta_{k+1}}=$ id for all $t \in[0,1]$. We define $h^{*}(t, u) \in \mathbf{C}([0,1] \times A, E)$ by $h^{*}(t, u)=h\left(t, \eta\left(T_{1}, u\right)\right)$. Then $h^{*}(t, u)$ is odd in $u \in A$ and $\left.h^{*}(t, \cdot)\right|_{\partial \Theta_{k+1}}=$ id for all $t \in[0,1]$. Consequently, $h^{*}(1, A) \cap S_{0}(k) \neq \emptyset$ since $A \in \mathcal{L}$. From this, it follows that $\eta\left(T_{1}, A\right) \in \mathcal{L}$. But by (3), $\eta\left(T_{1}, A\right) \cap \mathcal{S}^{*} \subset G^{C^{*}-\varepsilon_{1} / 4}$, which contradicts the definition of $C^{*}$.

By means of the next steps, we shall obtain information about the Morse index. Step 2. In this step and the next steps 3-5, we assume that $\mathcal{K}_{C^{*}} \cap \mathcal{S}^{*}$ contains finitely many nondegenerate critical points. We first prepare the way by stating some properties: for any $u \in \mathcal{K}_{C^{*}} \cap \mathcal{S}^{*}$ with $\operatorname{Morse}(u)=m^{*}$, we will show that we can always find a closed neighborhood $N_{1}(u)$ of $u$ such that $N_{1}(u) \cap \partial \Theta_{k+1}=\emptyset$ and 
find a subset $N_{2}(u)$ such that $N_{2}(u) \subset N_{1}(u) \subset \mathcal{S}^{*}$ where $N_{2}(u)$ is homeomorphic to a ball $B_{m^{*}}$ of $\mathbf{R}^{m^{*}}$. To show these properties, we let

$$
\mathcal{K}_{C^{*}} \cap \mathcal{S}^{*}=\left\{u_{1}, \ldots, u_{m}\right\} \cup\left\{-u_{1}, \ldots,-u_{m}\right\} .
$$

Since $C^{*} \geqq-\Lambda_{0}>>\sup _{u \in \partial \Theta_{k+1}} G$ and $G\left( \pm u_{i}\right)=C^{*}$ for all $i=1, \ldots, m$, we may find a $\delta>0$ such that $B_{\delta}\left( \pm u_{i}\right) \cap \partial \Theta_{k+1}=\emptyset, i=1,2, \ldots, m$; where $B_{\delta}\left( \pm u_{i}\right):=\left\{u \in E:\left\|u-\left( \pm u_{i}\right)\right\| \leqq \delta\right\}$. For each $u_{i}$ or $-u_{i}$, there exist closed subspaces of $E, E^{-}, E^{+}=\left(E^{-}\right)^{\perp}$ such that both subspaces are invariant under $G^{\prime \prime}\left(u_{i}\right) ; G^{\prime \prime}\left(u_{i}\right)$ is negative (positive) definite on $E^{-}\left(E^{+}\right)$. We write $z \in E$ as $z=z^{+}+z^{-}$with $z^{ \pm} \in E^{ \pm}$. By the Morse Lemma, there exists a Lipschitz homeomorphism $H_{i}$ from a neighborhood $U_{i}(0)$ of 0 in $E$ onto a neighborhood $U\left(u_{i}\right)$ of $u_{i}$ in $E$ with $H_{i}(0)=u_{i}$ such that

$$
G\left(H_{i}(z)\right)=G\left(u_{i}\right)+\left\|z^{+}\right\|^{2}-\left\|z^{-}\right\|^{2}, \quad \forall z \in U_{i}(0) .
$$

Obviously, $-H_{i}$ is a Lipschitz homeomorphism from the neighborhood $U_{i}(0)$ of 0 onto a neighborhood $-U\left(u_{i}\right)=U\left(-u_{i}\right)$ of $-u_{i}$ in $E$ with $-H_{i}(0)=-u_{i}$ and

$$
G\left(-H_{i}(z)\right)=G\left(-u_{i}\right)+\left\|z^{+}\right\|^{2}-\left\|z^{-}\right\|^{2}, \quad \forall z \in U_{i}(0) .
$$

Let $B_{i}^{-}$and $B_{i}^{+}$denote the closed ball of radius $r_{i}^{-}>0$ and $r_{i}^{+}>0$ centered at 0 in $E^{-}$and $E^{+}$respectively. Choose numbers $r_{i}^{-}$and $r_{i}^{+}$so small that $2 B_{i}^{-} \oplus B_{i}^{+}$ is contained in the domain $U_{i}(0)$ of $H_{i}$. We also assume that $4\left(r_{i}^{-}\right)^{2}<\left(r_{i}^{+}\right)^{2}$ for $i=1,2, \ldots, m$. We define

$$
D_{i}=H_{i}\left(B_{i}^{-} \oplus B_{i}^{+}\right), \quad D_{i}^{\prime}=H_{i}\left(2 B_{i}^{-} \oplus B_{i}^{+}\right), \quad i=1, \ldots, m .
$$

We may choose $r_{i}^{-}, r_{i}^{+}, \delta$ small enough such that

$$
\pm D_{i} \subset \pm D_{i}^{\prime} \subset B_{\delta}\left( \pm u_{i}\right) \subset \pm U\left(u_{i}\right) \subset \mathcal{S}^{*}, \quad i=1, \ldots, m .
$$

We may assume that $\left\{ \pm D_{i}\right\}_{i=1}^{m}$ are disjoint from each other. The same is true of $\left\{ \pm D_{i}^{\prime}\right\}_{i=1}^{m},\left\{B_{\delta}\left( \pm u_{i}\right)\right\}_{i=1}^{m}$ and $\left\{ \pm U\left(u_{i}\right)\right\}_{i=1}^{m}$. Let $\phi: \mathbf{R} \rightarrow[0,1]$ be a Lipschitz function such that $\phi(t)=0$ for $t \leqq 0$ and $\phi(t)=1$ for $t \geqq 1$. Inspired by Lazer and Solimini [16], we define $h: \mathbf{R} \times E \rightarrow E$ by

$$
\begin{aligned}
& h(t, z) \\
& \quad=\left\{\begin{array}{l}
z \\
\text { for } z \notin\left(\cup_{i=1}^{m} D_{i}^{\prime}\right) \cup\left(\cup_{i=1}^{m}\left(-D_{i}^{\prime}\right)\right), \\
H_{1}\left[t \phi\left(\frac{\left\|H_{1}^{-1}(z)^{-}\right\|}{r_{1}^{-}}-1\right) H_{1}^{-1}(z)^{+}+(1-t) H_{1}^{-1}(z)^{+}+H_{1}^{-1}(z)^{-}\right], \\
\text {for } z \in D_{1}^{\prime}, \\
-H_{1}\left[t \phi\left(\frac{\left\|H_{1}^{-1}(-z)^{-}\right\|}{r_{1}^{-}}-1\right) H_{1}^{-1}(-z)^{+}+(1-t) H_{1}^{-1}(-z)^{+}+H_{1}^{-1}(-z)^{-}\right], \\
\text {for } z \in-D_{1}^{\prime}, \\
\cdots \ldots \\
H_{m}\left[t \phi\left(\frac{\left\|H_{m}^{-1}(z)^{-}\right\|}{r_{m}^{-}}-1\right) H_{m}^{-1}(z)^{+}+(1-t) H_{m}^{-1}(z)^{+}+H_{m}^{-1}(z)^{-}\right], \\
\text {for } z \in D_{m}^{\prime}, \\
-H_{m}\left[t \phi\left(\frac{\left\|H_{m}^{-1}(-z)^{-}\right\|}{r_{m}^{-}}-1\right) H_{m}^{-1}(-z)^{+}+(1-t) H_{m}^{-1}(-z)^{+}+H_{m}^{-1}(-z)^{-}\right], \\
\text {for } z \in-D_{m}^{\prime} .
\end{array}\right.
\end{aligned}
$$


Then $h(t, z)$ is odd in $z \in E$ and continuous in

$$
E \backslash\left(\cup_{i=1}^{m}\left(-H_{i}\left(2 B_{i}^{-} \oplus \partial B_{i}^{+}\right) \cup H_{i}\left(2 B_{i}^{-} \oplus \partial B_{i}^{+}\right)\right)\right) .
$$

Choose $0<\varepsilon<\min _{i=1, \ldots, m}\left\{\left(r_{i}^{+}\right)^{2}-4\left(r_{i}^{-}\right)^{2}\right\}:=r^{*}$. Note that for any

$$
u \in \cup_{i=1}^{m}\left(\left(-H_{i}\left(2 B_{i}^{-} \oplus \partial B_{i}^{+}\right) \cup H_{i}\left(2 B_{i}^{-} \oplus \partial B_{i}^{+}\right)\right)\right),
$$

say, $u \in H_{i}\left(2 B_{i}^{-} \oplus \partial B_{i}^{+}\right)$, then $u=H_{i}(w), w=w^{+}+w^{-} \in 2 B_{i}^{-} \oplus \partial B_{i}^{+}$with $\left\|w^{+}\right\|=r_{i}^{+},\left\|w^{-}\right\| \leqq 2 r_{i}^{-}$, it follows that

$$
G(u)=G\left(H_{i}(w)\right)=C^{*}+\left\|w^{+}\right\|^{2}-\left\|w^{-}\right\|^{2} \geqq C^{*}+\left(r_{i}^{+}\right)^{2}-4\left(r_{i}^{-}\right)^{2}>C^{*}+\varepsilon .
$$

We observe that

$$
G^{C^{*}+\varepsilon} \subset E \backslash \cup_{i=1}^{m}\left(\left(-H_{i}\left(2 B_{i}^{-} \oplus \partial B_{i}^{+}\right) \cup H_{i}\left(2 B_{i}^{-} \oplus \partial B_{i}^{+}\right)\right)\right)
$$

for all $0<\varepsilon<r^{*}$. Furthermore,

$-h(0, z)=z$ for all $z \in E$;

- $h(t, z)=z$ for all $z \notin\left(\cup_{i=1}^{m} B_{\delta}\left(u_{i}\right)\right) \cup\left(\cup_{i=1}^{m} B_{\delta}\left(-u_{i}\right)\right)$;

- $\frac{\mathrm{d} G(h(t, z))}{\mathrm{d} t} \leqq 0$, this is, $G(h(t, z))$ is non-increasing in $t$ for any fixed $z \in E$.

Take $M_{i}=H_{i}\left(\frac{1}{3} B_{i}^{-} \oplus \frac{1}{3} B_{i}^{+}\right), i=1,2, \ldots, m$. Then by (5) $M_{i} \subset D_{i} \subset D_{i}^{\prime} \subset \mathcal{S}^{*}$. Define

$$
N_{2}\left(u_{i}\right)=h\left(1, G^{C^{*}+\varepsilon}\right) \cap M_{i} .
$$

Then $N_{2}\left(u_{i}\right) \subset N_{1}\left(u_{i}\right):=D_{i} \subset \mathcal{S}^{*}$. Note that $h$ is continuous on $G^{C^{*}+\varepsilon}$ by (7) and (8). For $w \in N_{2}\left(u_{i}\right)$, then $w \in M_{i}, w=H_{i}(v), v=H_{i}^{-1}(w)=v^{+} \oplus v^{-}, v^{ \pm} \in$ $\frac{1}{3} B_{i}^{ \pm} ; w$ is in the neighborhood $U\left(u_{i}\right)$ of $u_{i}$. On the other hand, $w=h(1, x), x \in$ $G^{C^{*}+\varepsilon}$. By (8), we see that

$$
x \in E \backslash \cup_{i=1}^{m}\left(\left(-H_{i}\left(2 B_{i}^{-} \oplus \partial B_{i}^{+}\right) \cup H_{i}\left(2 B_{i}^{-} \oplus \partial B_{i}^{+}\right)\right)\right) .
$$

If $x \notin\left(\cup_{i=1}^{m} D_{i}^{\prime}\right) \cup\left(\cup_{i=1}^{m}\left(-D_{i}^{\prime}\right)\right)$, then $w=h(1, x)=x \in M_{i} \subset D_{i}^{\prime}$, a contradiction. That is, there must exist some $D_{k_{0}}^{\prime}\left(\right.$ or $\left.-D_{k_{0}}^{\prime}\right)$ such that $x \in D_{k_{0}}^{\prime}\left(\right.$ or $\left.x \in-D_{k_{0}}^{\prime}\right)$ and hence

$$
\begin{aligned}
w & =h(1, x) \\
& =H_{k_{0}}\left(\phi\left(\frac{\left\|H_{k_{0}}^{-1}(x)^{-}\right\|}{r_{k_{0}}^{-}}-1\right) H_{k_{0}}^{-1}(x)^{+}+H_{k_{0}}^{-1}(x)^{-}\right)
\end{aligned}
$$

or respectively,

$$
\begin{aligned}
w & =h(1, x) \\
& =-H_{k_{0}}\left(\phi\left(\frac{\left\|H_{k_{0}}^{-1}(-x)^{-}\right\|}{r_{k_{0}}^{-}}-1\right) H_{k_{0}}^{-1}(-x)^{+}+H_{k_{0}}^{-1}(-x)^{-}\right) .
\end{aligned}
$$


It implies that $w$ is in the neighborhood of $u_{k_{0}}$ (or $\left.-u_{k_{0}}\right)$. Then we must have $w \in U\left(u_{k_{0}}\right)$ and $i=k_{0}$ and

$$
w=h(1, x)=H_{i}\left(\phi\left(\frac{\left\|H_{i}^{-1}(x)^{-}\right\|}{r_{i}^{-}}-1\right) H_{i}^{-1}(x)^{+}+H_{i}^{-1}(x)^{-}\right) .
$$

Then $v^{+}+v^{-}=H_{i}^{-1}(w)$ satisfying

$$
v^{-}=H_{i}^{-1}(w)^{-}=\left(H_{i}^{-1}(x)\right)^{-}, \quad\left\|v^{-}\right\|=\left\|H_{i}^{-1}(x)^{-}\right\| \leqq \frac{r_{i}^{-}}{3} ;
$$

it follows by the definition of $\phi$ that $v^{+}=\phi\left(\frac{\left\|H_{i}^{-1}(x)^{-}\right\|}{r_{i}^{-}}-1\right) H_{i}^{-1}(x)^{+}=0$. So $w=H_{i}\left(v^{-}\right)$with $v^{-} \in \frac{1}{3} B_{i}^{-}$. Moreover,

$$
G(w)=G\left(u_{i}\right)-\left\|v^{-}\right\|^{2} \leqq C^{*} .
$$

These arguments imply that

$$
N_{2}\left(u_{i}\right)=h\left(1, G^{C^{*}+\varepsilon}\right) \cap M_{i} \subset G^{C^{*}} .
$$

In particular, if $G(w)=C^{*}$ for $w \in h\left(1, G^{C^{*}+\varepsilon}\right) \cap M_{i} \cap G^{-1}\left(C^{*}\right)$, then by (10) $v^{-}=0$ and then $w=H_{i}\left(v^{-}\right)=H_{i}(0)=u_{i}$. Therefore,

$$
h\left(1, G^{C^{*}+\varepsilon}\right) \cap M_{i} \cap G^{-1}\left(C^{*}\right) \cap \mathcal{S}^{*}=\left\{u_{i}\right\} .
$$

So, for all $w \in N_{2}\left(u_{i}\right)$ we see that $w=H_{i}\left(v^{-}\right), v^{-} \in \frac{1}{3} B_{i}^{-}, H_{i}^{-1}(w)=v^{-}$. Conversely, for any $v^{-} \in \frac{1}{3} B_{i}^{-}$we have $H_{i}\left(v^{-}\right) \in M_{i}=H_{i}\left(\frac{1}{3} B_{i}^{-} \oplus \frac{1}{3} B_{i}^{+}\right) \subset D_{i} \subset D_{i}^{\prime}$. Let $z=H_{i}\left(v^{-}\right)$, then $v^{-}=H_{i}^{-1}(z)$ and hence

$$
\begin{aligned}
& h(1, z) \\
& =\left.H_{i}\left(t \phi\left(\frac{\left\|H_{i}^{-1}(z)^{-}\right\|}{r_{i}^{-}}-1\right) H_{i}^{-1}(z)^{+}+(1-t) H_{i}^{-1}(z)^{+}+H_{i}^{-1}(z)^{-}\right)\right|_{t=1} \\
& =H_{i}\left(H_{i}^{-1}(z)^{-}\right) \\
& =H_{i}\left(v^{-}\right) \\
& =z .
\end{aligned}
$$

Moreover, $G(z)=G\left(H_{i}\left(v^{-}\right)\right)=C^{*}-\left\|v^{-}\right\|^{2} \leqq C^{*}$, that is, $z=h(1, z) \in$ $h\left(1, G^{C^{*}+\varepsilon}\right)$ and $H_{i}\left(v^{-}\right) \in M_{i} \cap h\left(1, G^{C^{*}+\varepsilon}\right)=N_{2}\left(u_{i}\right)$ (cf. (9)). Summing up, we obtain that

$$
N_{2}\left(u_{i}\right) \text { is homeomorphic to the ball } \frac{1}{3} B_{i}^{-} \text {. }
$$

If we choose

$$
M_{i}^{*}=H_{i}\left(\frac{1}{4} B_{i}^{-} \oplus \frac{1}{4} B_{i}^{+}\right), \quad i=1,2, \ldots, m,
$$


we may prove analogously that

$$
N_{2}^{*}\left(u_{i}\right):=h\left(1, G^{C^{*}+\varepsilon}\right) \cap M_{i}^{*} \text { is homeomorphic to the ball } \frac{1}{4} B_{i}^{-} .
$$

Now we choose

$$
\begin{gathered}
N_{3}\left(u_{i}\right):=h\left(1, G^{C^{*}+\varepsilon}\right) \cap \partial M_{i}, \\
M_{i}^{\prime}=H_{i}\left(\frac{2}{3} B_{i}^{-} \oplus \frac{2}{3} B_{i}^{+}\right), \quad i=1,2, \ldots, m,
\end{gathered}
$$

then similar to (10)-(12), we may show that

$$
\begin{aligned}
& h\left(1, G^{C^{*}+\varepsilon}\right) \cap M_{i}^{\prime} \subset G^{C^{*}} ; \\
& h\left(1, G^{C^{*}+\varepsilon}\right) \cap M_{i}^{\prime} \cap G^{-1}\left(C^{*}\right) \cap \mathcal{S}^{*}=\left\{u_{i}\right\} ;
\end{aligned}
$$

and, by (5)-(6), $\pm M_{i}^{*} \subset \pm M_{i} \subset \pm M_{i}^{\prime} \subset \pm D_{i} \subset \pm D_{i}^{\prime} \subset B_{\delta}\left( \pm u_{i}\right) \subset \mathcal{S}^{*}$.

Step 3. Finding another $A^{*} \in \mathcal{L}$ which has properties stated in Step 4.

Given two neighborhoods $\mathcal{O}_{1} \subset \mathcal{O}$ of $\mathcal{K}_{C^{*}}$, we may then find an odd and continuous descending flow $\eta_{0}:[0,1] \times E \rightarrow E$ such that $\eta_{0}(t, u)=u$ for all $u \in \partial \Theta_{k+1}$ and all $u$ with $\left|G(u)-C^{*}\right| \geqq 2 \varepsilon ; \eta_{0}(1, u)=u$ for all $u \in \mathcal{O}_{1}$. In particular,

$$
\eta_{0}\left(1, G^{C^{*}+\varepsilon} \backslash \mathcal{O}\right) \subset G^{C^{*}-\varepsilon}
$$

This essentially comes from the proof of Theorem A.4 in [19]. Moreover, we may require that $\eta_{0}\left(t, \mathcal{D}^{*}\right) \subset \mathcal{D}^{*}$ for all $t \in[0,1]$, since the odd pseudo-gradient vector field $Y(u)=u-L_{0}(u)$ satisfying $L_{0}\left( \pm \mathcal{D}_{\mu_{0}}\right) \subset \pm \mathcal{D}_{\mu_{0}}$ (this is done in [22,26]). We may assume that $\mathcal{O}$ has two parts: $\mathcal{O}_{p n} \cup \mathcal{O}_{s}$, where $\mathcal{O}_{p n}$ is the neighborhood of all negative or positive critical points at level $C^{*}$ such that $\mathcal{O}_{p n} \subset \mathcal{D}^{*}$, and $\mathcal{O}_{s}$ is the neighborhood of all sign-changing critical points at level $C^{*}$. Hence,

$$
G^{C^{*}+\varepsilon} \backslash \mathcal{O}_{s} \subset\left(G^{C^{*}+\varepsilon} \backslash \mathcal{O}\right) \cup \mathcal{D}^{*}, \quad \eta_{0}\left(1, G^{C^{*}+\varepsilon} \backslash \mathcal{O}_{s}\right) \subset G^{C^{*}-\varepsilon} \cup \mathcal{D}^{*} .
$$

Let

$$
\begin{aligned}
F_{0} & =\mathcal{S}^{*} \backslash\left(\cup_{i=1}^{m} M_{i}^{\prime}\right) \cup\left(\cup_{i=1}^{m}\left(-M_{i}^{\prime}\right)\right), \\
G_{0} & =\left(\cup_{i=1}^{m} M_{i}\right) \cup\left(\cup_{i=1}^{m}\left(-M_{i}\right)\right) \subset \mathcal{S}^{*} .
\end{aligned}
$$

In particular, we choose

$$
\mathcal{O}_{s}:=\left(\cup_{i=1}^{m} \operatorname{int} M_{i}^{\prime}\right) \cup\left(\cup_{i=1}^{m} \operatorname{int}\left(-M_{i}^{\prime}\right)\right)
$$

and

$$
\left(\cup_{i=1}^{m} M_{i}^{*}\right) \cup\left(\cup_{i=1}^{m}\left(-M_{i}^{*}\right)\right) \subset \mathcal{O}_{1} .
$$

Then

$$
\eta_{0}\left(1, G^{C^{*}+\varepsilon} \cap F_{0}\right) \subset G^{C^{*}-\varepsilon} \cup \mathcal{D}^{*}
$$


Now we choose $A \in \mathcal{L}$ such that

$$
\sup _{A \cap \mathcal{S}^{*}} G \leqq C^{*}+\varepsilon .
$$

Now we let

$$
A^{*}:=\eta_{0}(1, h(1, A)) \text {. }
$$

We show that $A^{*} \in \mathcal{L}$. Obviously, $\partial \Theta_{k+1} \subset A^{*}, \sup G\left(A^{*}\right) \leqq C_{k+1}^{* *}$. Take any $h^{*}$ satisfying the assumptions of Definition 1 , that is, $h^{*} \in \mathbf{C}\left([0,1] \times A^{*}, E\right)$ satisfying

- $h^{*}(t, u)$ is odd in $u$;

- $h^{*}(t, u)=u$ for all $u \in \partial \Theta_{k+1}$.

Let

$$
f^{*}=h^{*}\left(t, \eta_{0}(1, h(1, u))\right), \quad u \in A .
$$

Then $f^{*}(t, u)$ is odd in $u ; f^{*}(t, u)=u$, for all $u \in \partial \Theta_{k+1}$. Hence, $f^{*}(1, A) \cap$ $S_{0}(k) \neq \emptyset$. That is, $h^{*}\left(1, A^{*}\right) \cap S_{0}(k) \neq \emptyset$. Therefore, $A^{*} \in \mathcal{L}$.

Step 4. We now show that

$$
\sup _{A^{*} \cap \mathcal{S}^{*}} G=C^{*}
$$

and

$$
G(u)<C^{*}, \quad \forall u \in\left(A^{*} \backslash \mathcal{K}_{C^{*}}\right) \cap \mathcal{S}^{*}
$$

In particular, there exists $u \in \mathcal{K}_{C^{*}} \cap \mathcal{S}^{*} \cap A^{*}$.

Indeed, for any $u \in A^{*} \cap \mathcal{S}^{*}$, write $u=\eta_{0}(1, v) \in \mathcal{S}^{*}$, then $v \in \mathcal{S}^{*}$ and $v=h(1, a)$ with $a \in \mathcal{S}^{*} \cap A$.

If $v \in F_{0}$ then $G(v)=G(h(1, a)) \leqq G(a) \leqq C^{*}+\varepsilon$. It follows that $v \in$ $F_{0} \cap G^{C^{*}+\varepsilon}$. Hence, $u=\eta_{0}(1, v) \in G^{C^{*}-\varepsilon} \cup \mathcal{D}^{*}$ by (20). Then, $u=\eta_{0}(1, v) \in$ $G^{C^{*}-\varepsilon}, G(u)<C^{*}, u \notin \mathcal{K}_{C^{*}}$.

If $v \notin F_{0}$, then $v \in\left(\cup_{i=1}^{m} M_{i}^{\prime}\right) \cup\left(\cup_{i=1}^{m}\left(-M_{i}^{\prime}\right)\right)$. Say $v \in M_{i}^{\prime}$ for some $i$. Since $v=h(1, a)$ with $a \in \mathcal{S}^{*}$ and $A \cap \mathcal{S}^{*} \subset G^{C^{*}+\varepsilon}$ by $(21)$, we have that $a \in G^{C^{*}+\varepsilon}$, $v=h(1, a) \in h\left(1, G^{C^{*}+\varepsilon}\right)$. Then $v \in M_{i}^{\prime} \cap h\left(1, G^{C^{*}+\varepsilon}\right)$, which implies by (17) that $G(v) \leqq C^{*}, G(u)=G\left(\eta_{0}(1, v)\right) \leqq G(v) \leqq C^{*}$. If $G(v)=C^{*}$, by (18) we have that $v=u_{i}$, hence $u=v=u_{i}$. If $G(v)<C^{*}$, then we see that $G(u)<c$. On the other hand, if $G(v)<C^{*}$ for all such $v$, then $G(u)<C^{*}$ for all $u \in A^{*} \cap \mathcal{S}^{*}$, which contradicts the definition of $C^{*}$ since $A^{*} \in \mathcal{L}$. These arguments imply that we must have a $v \in M_{i}^{\prime} \cap h\left(1, G^{C^{*}+\varepsilon}\right)$ such that $G(v)=C^{*}$ and therefore $v=u_{i}$, hence, $u=\eta_{0}(1, v)=\eta_{0}\left(1, u_{i}\right)=u_{i}$. That is, we have found a sign-changing critical point $u_{i}$ in $A^{*} \cap \mathcal{S}^{*}$ such that $G(u)=C^{*}$. This completes the step.

Step 5. We show that there is an $u \in \mathcal{K}_{C^{*}} \cap \mathcal{S}^{*} \cap A^{*}$ such that Morse $(u) \geqq k$. Note that $\mathcal{K}_{C^{*}} \cap \mathcal{S}^{*}=\left\{u_{1}, u_{2}, \ldots, u_{m}\right\} \cup\left\{-u_{1},-u_{2}, \ldots,-u_{m}\right\}$ (see (4)). By negation we assume that

$$
\operatorname{Morse}\left( \pm u_{i}\right)<k, \quad i=1,2, \ldots, m \text {. }
$$


Next, we show that

$$
A^{* *}:=A^{*} \backslash\left(\left(\cup_{i=1}^{m} \operatorname{int} M_{i}^{*}\right) \cup\left(\cup_{i=1}^{m} \operatorname{int}\left(-M_{i}^{*}\right)\right)\right) \in \mathcal{L} .
$$

If (26) were true, then we would have a contradiction to (23)-(24). Thus, (25) must be not true and the proof for the non-degenerate case of the Theorem is finished.

First of all, we keep in mind that $\partial \Theta_{k+1} \subset A^{* *}$ and $A^{* *} \subset A^{*} \subset G^{C_{k+1}^{* *}}$. Now by negation, if (26) does not hold, we find a $g$ such that $g \in \mathbf{C}\left([0,1] \times A^{* *}, E\right)$ satisfying: $g(t, u)$ is odd in $u ; g(t, u)=u$ for all $u \in \partial \Theta_{k+1}$, but

$$
g\left[1, A^{* *}\right] \cap S_{0}(k)=\emptyset .
$$

Note that $A^{* *}$ is compact. Since $A^{*} \cap M_{i}^{*} \subset A^{*} \cap \mathcal{S}^{*}$, we have

$$
\begin{aligned}
A^{*} \cap M_{i}^{*} & =\eta_{0}(1, h(1, A)) \cap M_{i}^{*} \\
& \subset \eta_{0}(1, h(1, A)) \cap \mathcal{S}^{*} \\
& \subset \eta_{0}\left(1, h(1, A) \cap \mathcal{S}^{*}\right) \cap \mathcal{S}^{*} \\
& \subset \eta_{0}\left(1, h\left(1, A \cap \mathcal{S}^{*}\right) \cap \mathcal{S}^{*}\right) \cap \mathcal{S}^{*} \\
& \subset \eta_{0}\left(1, h\left(1, G^{C^{*}+\varepsilon}\right) \cap \mathcal{S}^{*}\right) \cap \mathcal{S}^{*} \quad(\text { by }(21)) \\
& \subset \eta_{0}\left(1, h\left(1, G^{C^{*}+\varepsilon}\right) \cap \mathcal{S}^{*}\right) .
\end{aligned}
$$

Since (19) implies that $\left.\eta_{0}(1, \cdot)\right|_{M_{i}^{*}}=\mathbf{i d}$ for $i=1, \ldots, m$ and $\eta_{0}(1, \cdot)$ is a homeomorphism, we have

$$
A^{*} \cap M_{i}^{*}=\left.\eta_{0}^{-1}(1, \cdot)\right|_{A^{*} \cap M_{i}^{*}} \subset h\left(1, G^{C^{*}+\varepsilon}\right) \cap \mathcal{S}^{*} \subset h\left(1, G^{C^{*}+\varepsilon}\right),
$$

therefore, $A^{*} \cap M_{i}^{*} \subset h\left(1, G^{C^{*}+\varepsilon}\right) \cap M_{i}^{*}$. Recall (14), we see that $h\left(1, G^{C^{*}+\varepsilon}\right) \cap$ $M_{i}^{*}$ is homeomorphic to the ball $\frac{1}{4} B_{i}^{-}$, hence we obtain that $A^{*} \cap M_{i}^{*}$ is homeomorphic to a compact subset of $\mathbf{R}^{M \text { orse }\left(u_{i}\right)}$. We now observe that $g_{i}=g:[0,1] \times$ $\left(A^{*} \cap \partial M_{i}^{*}\right) \rightarrow E$ can be extended to

$$
g_{i}:\left([0,1] \times\left(A^{*} \cap \partial M_{i}^{*}\right)\right) \cup\left(\{0\} \times\left(A^{*} \cap M_{i}^{*}\right)\right) \rightarrow E
$$

Let $\tilde{g}_{i}(1, \cdot)$ be the extension of $g_{i}(1, \cdot)$ from the domain $A^{*} \cap \partial M_{i}^{*}$ to the domain $A^{*} \cap M_{i}^{*}$. For each $u \in A^{*} \cap M_{i}^{*}$, we write $\tilde{g}_{i}(1, u)=\tilde{g}_{i}(1, u)^{-}+\tilde{g}_{i}(1, u)^{+}$with $\tilde{g}_{i}(1, u)^{+} \in E_{k}^{\perp}, \tilde{g}_{i}(1, u)^{-} \in E_{k}$. Note that $\operatorname{dim} E_{k}=k$. Define

$$
\Omega_{i}=\left\{\begin{array}{ll}
u \in A^{*} \cap M_{i}^{*}: & \frac{\left\|\tilde{g}_{i}(1, u)^{+}\right\|\left\|\tilde{g}_{i}(1, u)^{+}\right\|_{\star}}{\left\|\tilde{g}_{i}(1, u)^{+}\right\|+\left\|\tilde{g}_{i}(1, u)^{+}\right\|_{\star}}=1, \\
\tilde{g}_{i}(1, u)^{-}=0
\end{array}\right\} .
$$

We see that $\tilde{g}_{i}\left(1, \Omega_{i}\right) \subset S_{0}(k)$. Since by (27),

$$
\tilde{g}_{i}\left(1, A^{*} \cap \partial M_{i}^{*}\right) \cap S_{0}(k)=g_{i}\left(1, A^{*} \cap \partial M_{i}^{*}\right) \cap S_{0}(k)=\emptyset,
$$


we see that $\Omega_{i} \cap\left(A^{*} \cap \partial M_{i}^{*}\right)=\emptyset, \Omega_{i}$ is a compact subset of $A^{*} \cap M_{i}^{*}$. We can find an $\varepsilon^{\prime}>$ small enough and define

$$
\Omega_{i}^{\varepsilon^{\prime}}=\left\{\begin{array}{ll} 
& \left\|\tilde{g}_{i}(1, u)^{-}\right\|<\varepsilon^{\prime} ; \\
u \in A^{*} \cap M_{i}^{*}: \begin{array}{l}
\left\|\tilde{g}_{i}(1, u)^{+}\right\|\left\|\tilde{g}_{i}(1, u)^{+}\right\|_{\star} \\
\end{array} \quad 1-\varepsilon^{\prime}<\frac{\tilde{g}_{i}(1, u)^{+}\|+\| \tilde{g}_{i}(1, u)^{+} \|_{\star}}{\| \tilde{g}^{\prime}},
\end{array}\right\}
$$

such that $\Omega_{i}^{\varepsilon^{\prime}} \cap\left(A^{*} \cap \partial M_{i}^{*}\right)=\varnothing$. Inspired by [20], we consider the mapping $\beta_{i}: \partial \Omega_{i}^{\varepsilon^{\prime}} \rightarrow\left(E_{k} \times \mathbf{R}\right) \backslash\{(0,1)\}$ by

$$
\beta_{i}(u)=\left(\tilde{g}_{i}(1, u)^{-}, \quad \frac{\left\|\tilde{g}_{i}(1, u)^{+}\right\|\left\|\tilde{g}_{i}(1, u)^{+}\right\|_{\star}}{\left\|\tilde{g}_{i}(1, u)^{+}\right\|+\left\|\tilde{g}_{i}(1, u)^{+}\right\|_{\star}}\right) .
$$

Since $\Omega_{i}^{\varepsilon^{\prime}}$ is a compact subset of $\mathbf{R}^{\operatorname{Morse}\left(u_{i}\right)}$ and $\operatorname{Morse}\left(u_{i}\right)<k<k+1=$ $\operatorname{dim}\left(E_{k} \times \mathbf{R}\right)$, it has an the extension $\tilde{\beta}_{i}: \Omega_{i}^{\varepsilon^{\prime}} \rightarrow\left(E_{k} \times \mathbf{R}\right) \backslash\{(0,1)\}$, so we write

$$
\tilde{\beta}_{i}(u)=\left(g_{i}^{*}(1, u), \rho(u)\right) .
$$

Then for any $u \in \partial \Omega_{i}^{\varepsilon^{\prime}}$ we have that $\tilde{\beta}_{i}(u)=\beta_{i}(u), g_{i}^{*}(1, u)=\tilde{g}_{i}(1, u)^{-}$;

$$
\rho(u)=\frac{\left\|\tilde{g}_{i}(1, u)^{+}\right\|\left\|\tilde{g}_{i}(1, u)^{+}\right\|_{\star}}{\left\|\tilde{g}_{i}(1, u)^{+}\right\|+\left\|\tilde{g}_{i}(1, u)^{+}\right\|_{\star}} .
$$

Define $T_{i}(u): A^{*} \cap M_{i}^{*} \rightarrow E$ as follows:

$$
T_{i}(u)= \begin{cases}g_{i}^{*}(1, u)+\rho(u) \frac{\tilde{g}_{i}(1, u)^{+}}{\frac{\left\|\tilde{g}_{i}(1, u)^{+}\right\|\left\|\tilde{g}_{i}(1, u)^{+}\right\|_{\star}}{\left\|\tilde{g}_{i}(1, u)^{+}\right\|+\left\|\tilde{g}_{i}(1, u)^{+}\right\|_{\star}},} & \text { for } u \in \Omega_{i}^{\varepsilon^{\prime}}, \\ \tilde{g}_{i}(1, u), & \text { for } u \notin \Omega_{i}^{\varepsilon^{\prime}} .\end{cases}
$$

On the other hand, if $u \notin \Omega_{i}^{\varepsilon^{\prime}}$, then $u \notin \Omega_{i}$, then either

$$
\frac{\left\|\tilde{g}_{i}(1, u)^{+}\right\|\left\|\tilde{g}_{i}(1, u)^{+}\right\|_{\star}}{\left\|\tilde{g}_{i}(1, u)^{+}\right\|+\left\|\tilde{g}_{i}(1, u)^{+}\right\|_{\star}} \neq 1
$$

or $\tilde{g}_{i}(1, u)^{-} \neq 0$. In this case, $T_{i}(u)=\tilde{g}_{i}(1, u)=\tilde{g}_{i}(1, u)^{-}+\tilde{g}_{i}(1, u)^{+} \notin S_{0}(k)$. Assume $u \in \Omega_{i}^{\varepsilon^{\prime}}$. If

$$
T_{i}(u)=g_{i}^{*}(1, u)+\rho(u) \frac{\tilde{g}_{i}(1, u)^{+}}{\frac{\left\|\tilde{g}_{i}(1, u)^{+}\right\|\left\|\tilde{g}_{i}(1, u)^{+}\right\|_{\star}}{\left\|\tilde{g}_{i}(1, u)^{+}\right\|+\left\|\tilde{g}_{i}(1, u)^{+}\right\|_{\star}}} \in S_{0}(k),
$$

we must have $g_{i}^{*}(1, u)=0$ and $\|\rho(u)\|=1$, which contradicts their definitions. Therefore,

$$
T_{i}\left(A^{*} \cap M_{i}^{*}\right) \cap S_{0}(k)=\emptyset .
$$


Let

$$
\bar{g}_{i}(t, u)= \begin{cases}g_{i}(t, u), & \text { for others } \\ T_{i}(u), & \text { for } t=1, u \in A^{*} \cap M_{i}^{*}\end{cases}
$$

Then $\bar{g}_{i}$ is the extension of $g_{i}$ from the domain $\left([0,1] \times A^{*} \cap \partial M_{i}^{*}\right) \cup(\{0\} \times$ $\left.A^{*} \cap M_{i}^{*}\right)$ to the domain $\left([0,1] \times A^{*} \cap \partial M_{i}^{*}\right) \cup\left(\{0,1\} \times A^{*} \cap M_{i}^{*}\right)$. By (29), we have

$$
\bar{g}_{i}\left(1, A^{*} \cap M_{i}^{*}\right) \cap S_{0}(k)=T_{i}\left(A^{*} \cap M_{i}^{*}\right) \cap S_{0}(k)=\emptyset .
$$

Next we show that

$$
\bar{g}_{i}(t, u):\left([0,1] \times\left(A^{*} \cap \partial M_{i}^{*}\right)\right) \cup\left(\{0,1\} \times\left(A^{*} \cap M_{i}^{*}\right)\right) \rightarrow E
$$

has an extension $\Psi_{i}^{*}:[0,1] \times\left(A^{*} \cap M_{i}^{*}\right) \rightarrow E$ such that

$$
\Psi_{i}^{*}\left(1, A^{*} \cap M_{i}^{*}\right) \cap S_{0}(k)=\emptyset .
$$

First we let $G_{i}(t, u):[0,1] \times\left(A^{*} \cap M_{i}^{*}\right) \rightarrow E$ be an extension of $\bar{g}_{i}(t, u)$. We write $G_{i}(t, u)=G_{i}(t, u)^{-}+G_{i}(t, u)^{+}$with $G_{i}(t, u)^{+} \in E_{k}^{\perp}$ and $G_{i}(t, u)^{-} \in E_{k}$. Define

$$
\Lambda_{i}:=\left\{(t, u) \in[0,1] \times\left(A^{*} \cap M_{i}^{*}\right): \begin{array}{l}
G_{i}(t, u)^{-}=0 \\
\frac{\left\|G_{i}(t, u)^{+}\right\|\left\|G_{i}(t, u)^{+}\right\|_{\star}}{\left\|G_{i}(t, u)^{+}\right\|+\left\|G_{i}(t, u)^{+}\right\|_{\star}}=1
\end{array}\right\} .
$$

Then $\Lambda_{i}$ is a compact subset of $\mathbf{R}^{\text {Morse }\left(u_{i}\right)+1}$ and by (30), we observe that

$$
\Lambda_{i} \cap\left(\{1\} \times\left(A^{*} \cap \partial M_{i}^{*}\right)\right)=\emptyset .
$$

As before, for $\varepsilon^{\prime}>0$ small enough, let

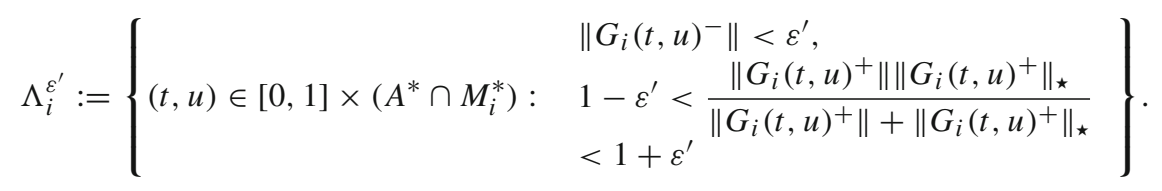

Consider the map $\Psi_{i}: \partial \Lambda_{i}^{\varepsilon^{\prime}} \rightarrow\left(E_{k} \times \mathbf{R}\right) \backslash\{(0,1)\}$ given by

$$
\Psi_{i}(t, u)=\left(G_{i}(t, u)^{-}, \frac{\left\|G_{i}(t, u)^{+}\right\|\left\|G_{i}(t, u)^{+}\right\|_{\star}}{\left\|G_{i}(t, u)^{+}\right\|+\left\|G_{i}(t, u)^{+}\right\|_{\star}}\right) .
$$

Since Morse $\left(u_{i}\right)+1<k+1, \Psi_{i}$ has an extension $\bar{\Psi}_{i}: \Lambda_{i}^{\varepsilon^{\prime}} \rightarrow\left(E_{k} \times \mathbf{R}\right) \backslash\{(0,1)\}$. We denote $\bar{\Psi}_{i}$ by $\bar{\Psi}_{i}(t, u)=\left(\bar{G}_{i}(t, u)^{-}, \xi(t, u)\right)$ with $\bar{G}_{i}(t, u)^{-} \in E_{k}$ and $\xi(t, u) \in \mathbf{R}$. Define $\Psi_{i}^{*}(t, u):[0,1] \times\left(A^{*} \cap M_{i}^{*}\right) \rightarrow E$ by

$$
\Psi_{i}^{*}(t, u)= \begin{cases}\bar{G}_{i}(t, u)^{-}+\xi(t, u) \frac{G_{i}(t, u)^{+}}{\frac{\left\|G_{i}(t, u)^{+}\right\|\left\|G_{i}(t, u)^{+}\right\|_{\star}}{\left\|G_{i}(t, u)^{+}\right\|+\left\|G_{i}(t, u)^{+}\right\|_{\star}}}, & \text { for }(t, u) \in \Lambda_{i}^{\varepsilon}, \\ G_{i}(t, u), & \text { for }(t, u) \notin \Lambda_{i}^{\varepsilon} .\end{cases}
$$


Then $\Psi_{i}^{*}$ from the domain $[0,1] \times\left(A^{*} \cap M_{i}^{*}\right)$ is an extension of $\bar{g}_{i}(t, u)$ such that (31) holds. Now, we define

$$
H(t, u)= \begin{cases}\Psi_{1}^{*}(t, u), & \text { for } u \in A^{*} \cap M_{1}^{*}, \\ -\Psi_{1}^{*}(t,-u), & \text { for } u \in A^{*} \cap\left(-M_{1}^{*}\right), \\ \ldots \ldots & \text { for } u \in A^{*} \cap M_{m}^{*}, \\ \Psi_{m}^{*}(t, u), & \text { for } u \in A^{*} \cap\left(-M_{m}^{*}\right), \\ -\Psi_{m}^{*}(t,-u), & \text { for } u \in A^{*} \backslash\left(\left(\cup_{i=1}^{m} \operatorname{int} M_{i}^{*}\right) \cup\left(\cup_{i=1}^{m} \operatorname{int}\left(-M_{i}^{*}\right)\right)\right) . \\ g(t, u), & \end{cases}
$$

Then $H(t, u)$ is odd in $u$ and $\left.H(t, u)\right|_{\partial \Theta_{k+1}}=\left.g(t, u)\right|_{\partial \Theta_{k+1}}=$ id, $\quad t \in[0,1]$. That is, $H$ satisfies the properties of Definition 1 with respect to $A^{*}$. But by (27) and (31), $H\left(1, A^{*}\right) \cap S_{0}(k)=\emptyset$, which contradicts the fact that $A^{*} \in \mathcal{L}$.

Step 6. To finish the proof of this theorem, we have only to consider the degenerate critical points of $\mathcal{K}_{C^{*}} \cap \mathcal{S}^{*}$. The idea is classical. But since we want to obtain sign-changing critical points, we must say more. We will apply the ideas of the Marino-Prodi type perturbation methods (see Marino and Prodi [18] and also Lazer and Solimini [16] and Viterbo [25]) and a simple modification of the arguments in Section 3 of Solimini [24].

Assume by negation that each critical point of $G$ in $\mathcal{K}_{C^{*}} \cap \mathcal{S}^{*}$ has an augmented Morse index less than $k$. Then for any $w \in \mathcal{K}_{C^{*}} \cap \mathcal{S}^{*}$, there is a decomposition of $E: E=E^{-}(w) \oplus E^{0}(w) \oplus E^{+}(w)$ and there exists a constant $\rho_{w}>0$ such that

$$
\begin{aligned}
& \left\langle G^{\prime \prime}(w) u, u\right\rangle \geqq \rho_{w}\|u\|^{2}, \quad \forall u \in E^{+}(w), \\
& \left\langle G^{\prime \prime}(w) u, u\right\rangle \leqq-\rho_{w}\|u\|^{2}, \quad \forall u \in E^{-}(w), \\
& \left\langle G^{\prime \prime}(w) u, u\right\rangle=0, \quad \forall u \in E^{0}(w),
\end{aligned}
$$

where

$$
\operatorname{dim} E^{-}(w)+\operatorname{dim} E^{0}(w)<k, \quad \forall w \in \mathcal{K}_{C^{*}} \cap \mathcal{S}^{*}
$$

By the Sard-Smale Theorem, we find regular values $C_{1}$ and $C_{2}$ of $G$ such that $C_{1}<C^{*}<C_{2}$. Let $M_{0}:=\mathcal{K}\left[C_{1}, C_{2}\right] \cap \mathcal{S}^{*}$. Then $M_{0}$ is compact and isolated from the rest of the critical points of $G$. Since $G$ is of $\mathbf{C}^{2}$, for each $w \in M_{0}$ we may find a small open ball $B_{w}$ centered at $w$ satisfying

$$
\left\|G^{\prime \prime}(w)-G^{\prime \prime}(u)\right\|<\rho_{w} / 2, \quad \forall u \in B_{w} .
$$

We may assume that $M_{0} \subset \cup_{i=1}^{m} B_{w_{i}}$. Define $\rho^{*}:=\frac{1}{2} \min \left\{\rho_{w_{1}}, \rho_{w_{2}}, \ldots, \rho_{w_{m}}\right\}$. By (32) and (34) we have

$$
\left\langle G^{\prime \prime}(v) u, u\right\rangle \geqq \rho^{*}\|u\|^{2}, \quad \forall v \in B_{w_{i}}, \quad \forall u \in E^{+}\left(w_{i}\right), \quad i=1, \ldots, m .
$$

Now by the Marino-Prodi-Solimini's perturbation methods mentioned above, for any $v>0$ small enough and a functional $G_{v} \in \mathbf{C}^{2}(E, \mathbf{R})$ such that 
(1) $U_{v}\left(M_{0}\right) \subset \cup_{i=1}^{m} B_{w_{i}}$, where $U_{v}\left(M_{0}\right):=\left\{u \in E: \operatorname{dist}\left(u, M_{0}\right)<v\right\}$,

(2) $G_{v}$ satisfies the (PS) condition,

(3) $G(u)=G_{v}(u)$ when $u \notin U_{v}\left(M_{0}\right)$,

(4) any critical point of $G_{v}$ in $U_{v}\left(M_{0}\right)$ is non-degenerate,

(5) $\left|G_{v}(u)-G(u)\right| \leqq v,\left\|G_{v}^{\prime}(u)-G^{\prime}(u)\right\| \leqq v,\left\|G_{v}^{\prime \prime}(u)-G^{\prime \prime}(u)\right\| \leqq v$ for all $u \in E$. In particular, we may let $G_{v}$ be an even functional since $G$ is also.

On the other hand, for the perturbed functional $G_{v}$, we still have conditions $\left(A_{2}\right)-\left(A_{3}\right)$ if $v$ small enough. To keep the flow-invariance, we write

$$
G_{v}^{\prime}(u)=G^{\prime}(u)-\theta(u)=u-\left(K_{G}(u)+\theta(u)\right):=u-K_{G}^{*}(u) .
$$

Since $\|\theta(u)\| \leqq v$ is small enough, we may suppose that $2 \theta(u) \in\left(-\mathcal{D}^{*} \cap \mathcal{D}^{*}\right)$. Then by assumption $\left(A_{1}\right)$ for $G$, we still have that $K_{G}^{*}\left( \pm \mathcal{D}\left(\mu_{0}\right)\right) \subset \pm \mathcal{D}(\mu) \subset$ $\pm \mathcal{D}\left(\mu_{0}\right)$ for another $\mu \in\left(0, \mu_{0}\right)$. That is, $\left(A_{1}\right)$ holds for $G_{\nu}$. Hence, we may apply the results of Step 1 to $G_{v}$ to conclude that $G_{v}$ has a sign-changing critical point at level $C_{v}^{*}$ defined as $C^{*}$. By item (5) above, we see that $C_{v}^{*} \in\left(C_{1}, C_{2}\right)$ since $v$ is very small. By item (3), we have that

$$
\mathcal{K}_{C_{v}^{*}}\left(G_{v}\right) \cap \mathcal{S}^{*}:=\left\{u \in E: G_{v}^{\prime}(u)=0, G_{v}(u)=C_{v}^{*}\right\} \cap \mathcal{S}^{*} \subset U_{v}\left(M_{0}\right)
$$

By item (4), $\mathcal{K}_{C_{v}^{*}}\left(G_{v}\right) \cap \mathcal{S}^{*}$ consists of non-degenerate critical points of $G_{v}$ and hence, is a finite set. Hence, by the results of steps $2-5, G_{v}$ has a sign-changing critical point $w_{0} \in \mathcal{K}_{C_{\nu}^{*}}\left(G_{\nu}\right) \cap \mathcal{S}^{*}$ whose Morse index is not less than $k$. Assume $w_{0} \in B_{w_{i}}$ for some $i$ with $1 \leqq i \leqq m$, then by item (5) and (35), we have

$$
\left\langle G_{v}^{\prime \prime}\left(w_{0}\right) u, u\right\rangle \geqq\left\langle G^{\prime \prime}\left(w_{0}\right) u, u\right\rangle-\frac{\rho^{*}}{2}\|u\|^{2} \geqq \frac{\rho^{*}}{2}\|u\|^{2}, \quad \forall u \in E^{+}\left(w_{i}\right) .
$$

By (33), we have codim $E^{+}\left(w_{i}\right)=\operatorname{dim} E^{-}\left(w_{i}\right)+\operatorname{dim} E^{0}\left(w_{i}\right)<k$. Therefore, the Morse index of $w_{0}$ is less than $k$. This is a contradiction which completes the proof of the Theorem 2 .

\section{Proof of Theorem 1}

We fix a $p_{0} \in\left(2,2^{*}\right)$ and choose a sequence $\left\{p_{n}\right\}_{n \in \mathbf{N}}$ in $\left(p_{0}, 2^{*}\right)$ such that $p_{n} \rightarrow 2^{*}$. Consider the functional

$$
G_{n, \lambda}(u)=\frac{1}{2}\|u\|^{2}-\frac{\lambda}{2} \int_{\Omega}|u|^{2} \mathrm{~d} x-\frac{1}{p_{n}} \int_{\Omega}|u|^{p_{n}} \mathrm{~d} x, \quad u \in H_{0}^{1}(\Omega),
$$

where $H_{0}^{1}(\Omega)$ is the usual Sobolev space with the inner product $\langle u, v\rangle=\int_{\Omega} \nabla u$. $\nabla v \mathrm{~d} x$ and the corresponding norm $\|u\|=\langle u, u\rangle^{1 / 2}$. Let $0<\lambda_{1}<\lambda_{2} \leqq \lambda_{3} \leqq$ $\cdots \leqq \lambda_{k} \leqq \cdots$ be the eigenvalues of $(-\Delta, \Omega)$ and $\phi_{k}(x)$ be the eigenfunction corresponding to $\lambda_{k}$. Denote $E_{k}:=\operatorname{span}\left\{\phi_{1}, \phi_{2}, \ldots, \phi_{k}\right\}$. For each $p_{n} \in\left(2,2^{*}\right)$, we let $\|\cdot\|_{\star}=\|\cdot\|_{p_{n}}$ (the usual $L^{p_{n}}$ norm). Then $\left(A_{0}\right)$ of Section 2 is satisfied. The gradient $G_{n, \lambda}^{\prime}$ is of the form $G_{n, \lambda}^{\prime}(u)=u-K_{n, \lambda}(u)$, where $K_{n, \lambda}: E \rightarrow E$ is a continuous operator. Let $\mathcal{P}:=\left\{u \in H_{0}^{1}(\Omega): u \geqq 0\right\}$. For each $\mu>0$, define $\mathcal{D}(\mu):=\{u \in$ $E: \operatorname{dist}(u, \mathcal{P})<\mu\}$. Set $\mathcal{D}^{*}=\mathcal{D}^{*}(\mu):=\mathcal{D}(\mu) \cup(-\mathcal{D}(\mu)), \quad \mathcal{S}^{*}=E \backslash \mathcal{D}^{*}(\mu)$. 
Then it is easy to check that $\left(A_{1}\right)$ is satisfied by $K_{n, \lambda}$, see for example [10, Lemma 2] (and also $[5,12,13,22])$. The assumptions $\left(A_{2}\right)-\left(A_{3}\right)$ are evidently true for $G_{n, \lambda}$. Let

$$
C_{k+1}^{* *}(n, \lambda):=\sup _{E_{k+1}} G_{n, \lambda}
$$

Lemma 4. There exists a constant $T_{1}>0$ independent of $k$ and $n$ such that

$$
C_{k+1}^{* *}(n, \lambda) \leqq T_{1} \lambda_{k+1}^{\frac{p_{0}}{2\left(p_{0}-2\right)}}
$$

Proof. This is standard. We include the proof here for completeness. The definition of $E_{k+1}$ implies that $\|u\|^{2} \leqq \lambda_{k+1}\|u\|_{2}^{2}$. Note that with $p_{n}>p_{0}$, we have $\|u\|_{p_{0}} \leqq D_{1}\|u\|_{p_{n}}$, where $D_{1}>0$ is a constant independent of $n$ and $k$. Therefore, $G_{n, \lambda}(u) \leqq \frac{1}{2}\|u\|^{2}-D_{2} \int_{\Omega}|u|^{p_{0}} \mathrm{~d} x+D_{3}$, where $D_{2}>0, D_{3}>0$ are constant, independent of $n$ and $k$. Since there is a constant $D_{4}>0$ such that $\|u\|_{2} \leqq D_{4}\|u\|_{p_{0}}$, therefore we may have $D_{5}>0$ such that $\|u\|^{p_{0}} \leqq D_{5} \lambda_{k+1}^{p_{0} / 2}\|u\|_{p_{0}}^{p_{0}}$ for all $u \in E_{k+1}$. Then

$$
\begin{aligned}
G_{n, \lambda}(u) & \leqq \frac{1}{2}\|u\|^{2}-D_{6} \lambda_{k+1}^{-p_{0} / 2}\|u\|^{p_{0}}+D_{3} \\
& \leqq D_{7} \lambda_{k+1}^{\frac{p_{0}}{2\left(p_{0}-2\right)}}+D_{3} \\
& \leqq T_{1} \lambda_{k+1}^{\frac{p_{0}}{2\left(p_{0}-2\right)}},
\end{aligned}
$$

where $D_{i}(i=1, \ldots, 7)$ and $T_{1}$ are positive constants independent of $k$ and $n$.

By Theorem 2, there is a sign-changing critical point $u^{*}(n, \lambda, k) \neq 0$ of $G_{n, \lambda}$ such that

$$
G_{n, \lambda}\left(u^{*}(n, \lambda, k)\right)=C^{*}(n, \lambda, k) \leqq C_{k+1}^{* *}(n, \lambda) \leqq T_{1} \lambda_{k+1}^{\frac{p_{0}}{2\left(p_{0}-2\right)}}
$$

and the augmented Morse index $m^{*}\left(u^{*}(n, \lambda, k)\right)$ of $u^{*}(n, \lambda, k)$ is $\geqq k$. We now must estimate the lower bound of $C^{*}(n, \lambda, k)$. By the proof of Theorem 2, for $G_{n, \lambda}$ we choose $\|\cdot\|_{\star}=\|\cdot\|_{p_{n}}$ and then

$$
\beta^{*}(u):=\beta_{n, \lambda}^{*}(u)=\left\{\begin{array}{cc}
\frac{\|u\|\|u\|_{p_{n}}}{\|u\|+\|u\|_{p_{n}}}, & u \neq 0, \\
0, & u=0
\end{array}\right.
$$

and $S_{0}(n, k):=\left\{u \in E_{k}^{\perp}: \beta_{n, \lambda}^{*}(u)=1\right\}$. Since $\|u\|_{p_{n}} \leqq \kappa_{1}\|u\|$ for all $u \in E$, where $\kappa_{1}$ is independent of $n$ and $k$. Then $\|u\|_{p_{n}} \leqq \kappa_{1}+1:=\alpha_{4}$ (as in Section 2) for all $u \in S_{0}(n, k)$. Recall $S(k):=S(n, k):=S_{0}(n, k) \cap G_{n, \lambda}^{C_{k+1}^{* *}(n, \lambda)}$. Then

$$
\|u\|^{2} \leqq \frac{\lambda_{1}}{\lambda_{1}-\lambda}\left(\left(\kappa_{1}+1\right)^{p_{n}}+2 C_{k+1}^{* *}(n, \lambda)\right), \quad \forall u \in S(n, k)
$$

Hence, as in Lemma 1, we find $\alpha_{5}>0$ such that such that $\|u\| \leqq \alpha_{5}$ for all $u \in$ $S(n, k)$. In particular, by (36)-(37), $\alpha_{5}>0$ is independent of $n$. Using Lemma 1 , 
there is a $\Lambda_{0}=\Lambda_{0}\left(\alpha_{5}\right)>0$ such that $\inf _{u \in S(n, k)} G_{n, \lambda} \geqq-\Lambda_{0}$, where $\Lambda_{0}$ is independent of $n$. By Theorem 2 and (36),

$$
C^{*}(n, \lambda, k) \in\left[-\Lambda_{0}, T_{1} \lambda_{k+1}^{\frac{p_{0}}{2\left(p_{0}-2\right)}}\right] .
$$

For the fixed $\lambda, k,\left\{u^{*}(n, \lambda, k)\right\}_{n \in \mathbf{N}}$ is a sequence of solutions to the following equation:

$$
-\Delta u=\lambda u+|u|^{p_{n}-2} u, \quad u \in H_{0}^{1}(\Omega),
$$

for $p_{n}$ varying in $\left[p_{0}, 2^{*}\right]$. By Lemma 4 , it is easy to check that $\left\{u^{*}(n, \lambda, k)\right\}_{n \in \mathbf{N}}$ is bounded in $H_{0}^{1}(\Omega)$. Here we insert the following proposition attributable to Devillanova and Solimini [14].

Proposition 1. (Uniform bound through concentration estimates) Let $N \geqq 7$ and $U$ be a bounded set in $H_{0}^{1}(\Omega)$ whose elements are solutions, for a fixed $\lambda>0$, to the problem

$$
-\Delta u=\lambda u+|u|^{p-2} u, \quad u \in H_{0}^{1}(\Omega),
$$

for $p$ varying in $\left[2,2^{*}\right]$. Then $U$ is uniformly bounded, that is, there exists a constant $C>0$ such that

$$
\sup _{u \in U} \sup _{x \in \Omega}|u(x)| \leqq C .
$$

By this proposition, we know that $\left\{u^{*}(n, \lambda, k)\right\}_{n \in \mathbf{N}}$ is uniformly bounded. Then, by standard compactness arguments we have a convergent subsequence having limit $u^{*}(\lambda, k)$ which is a solution of $(1)$ at level $C^{*}(\lambda, k)$. Since (1) has no solution with negative energy, we know that $C^{*}(\lambda, k) \geqq 0$. By (38),

$$
0 \leqq \lim _{n \rightarrow \infty} C^{*}(n, \lambda, k)=C^{*}(\lambda, k) \leqq T_{1} \lambda_{k+1}^{\frac{p_{0}}{2\left(p_{0}-2\right)}} .
$$

Moreover, we claim that $u^{*}(\lambda, k)$ is still sign-changing. Indeed, since $\left\{u^{*}(n\right.$, $\lambda, k)\}_{n \in \mathbf{N}}$ is a sequence of sign-changing solutions to (39), let

$$
u^{*}(n, \lambda, k)^{ \pm}:=\max \left\{ \pm u^{*}(n, \lambda, k), 0\right\}
$$

Then $\left\|u^{*}(n, \lambda, k)^{ \pm}\right\|^{2}=\lambda\left\|u^{*}(n, \lambda, k)^{ \pm}\right\|_{2}^{2}+\int_{\Omega}\left|u^{*}(n, \lambda, k)^{ \pm}\right|^{p_{n}} \mathrm{~d} x$. It follows that $\left\|u^{*}(n, \lambda, k)^{ \pm}\right\|^{2} \leqq \varepsilon_{0}\left\|u^{*}(n, \lambda, k)^{ \pm}\right\|_{p_{n}}^{p_{n}}$. Hence, $\left\|u^{*}(n, \lambda, k)^{ \pm}\right\| \geqq s_{0}>0$, where $\varepsilon_{0}, s_{0}$ are constants independent of $n$. This implies that the limit $u^{*}(\lambda, k)$ of the subsequence of $\left\{u^{*}(n, \lambda, k)\right\}_{n \in \mathbf{N}}$ is still sign-changing. Next, we have only to show that $\lim _{k \rightarrow \infty} C^{*}(\lambda, k)=\infty$. Otherwise, we assume that $\left\{C^{*}(\lambda, k)\right\}_{k \in \mathbf{N}}$ is bounded and then $\lim _{k \rightarrow \infty} C^{*}(\lambda, k)=c^{\prime}<\infty$. For any $k \in \mathbf{N}$ we may find an $n_{k}$ (we may assume $\left.n_{k}>k\right)$ such that $\left|C^{*}\left(n_{k}, \lambda, k\right)-C^{*}(\lambda, k)\right|<1 / k$. It follows that $\lim _{k \rightarrow \infty} C^{*}\left(n_{k}, \lambda, k\right)=\lim _{k \rightarrow \infty} C^{*}(\lambda, k)=c^{\prime}<\infty$. Since $u^{*}\left(n_{k}, \lambda, k\right)$ is a sign-changing critical point of $G_{n_{k}, \lambda}$ such that $G_{n_{k}, \lambda}\left(u^{*}\left(n_{k}, \lambda, k\right)\right)=C^{*}\left(n_{k}, \lambda, k\right)$ and the augmented Morse index $m^{*}\left(u^{*}\left(n_{k}, \lambda, k\right)\right)$ of $u^{*}\left(n_{k}, \lambda, k\right)$ is greater than or equal to $k$. Once again, we may show that $\left\{u^{*}\left(n_{k}, \lambda, k\right)\right\}$ is bounded in $H_{0}^{1}(\Omega)$ 
since $c^{\prime}<\infty$ is independent of $k$. Hence, by Proposition $1,\left\{u^{*}\left(n_{k}, \lambda, k\right)\right\}_{k \in \mathbf{N}}$ is uniformly bounded, hence the Morse index of $\left\{u^{*}\left(n_{k}, \lambda, k\right)\right\}_{k \in \mathbf{N}}$ is bounded. This contradiction is caused by the assumption that $\left\{C^{*}(\lambda, k)\right\}_{k \in \mathbf{N}}$ is bounded. This finishes the proof of Theorem 1.

Acknowledgments. W. Zou thanks Professor M. Ramos so much for many enlightening discussions when he was visiting CMAF (Lisboa, Portugal). RAmos' suggestions are very highly appreciated. Schechter is supported by NSF. Zou is supported by NSFC (10871109, 10571096) and the program of the Ministry of Education in China for NCET in Universities of China.

Open Access This article is distributed under the terms of the Creative Commons Attribution Noncommercial License which permits any noncommercial use, distribution, and reproduction in any medium, provided the original author(s) and source are credited.

\section{References}

1. Atkinson, F.V., Brézis, H., Peletier, L.A.: Nodal solutions of elliptic equations with critical Sobolev exponents. J. Differ. Equ. 85, 151-170 (1990)

2. BartsCH, T.: Critical point theory on partially ordered Hilbert spaces. J. Funct. Anal. 186, 117-152 (2001)

3. Bartsch, T., Chang, K.C., WAng, Z.-Q.: On the Morse indices of sign-changing solutions for nonlinear elliptic problems. Math. Z. 233, 655-677 (2000)

4. Bartsch, T., Maria Mecheletti, A., Pistoia, A.: On the existence and profile of nodal solutions of elliptic equations involving critical growth. Cal. Var. PDE. 26(3), 265-282 (2006)

5. Bartsch, T., Liu, Z., Weth, T.: Sign changing solutions of superlinear Schrödinger equations. Comm. Partial Differ. Equ. 29, 25-42 (2000)

6. Bartsch, T., Weth, T.: A note on additional properties of sign changing solutions to superlinear elliptic equations. Topol. Methods Nonlinear Anal. 22, 1-14 (2003)

7. Brézis, H., Nirenberg, L.: Positive solutions of nonlinear elliptic equations involving critical Sobolev exponents. Comm. Pure Appl. Math. 36, 437-477 (1983)

8. Cerami, G., Fortunato, D., Struwe, M.: Bifurcation and multiplicity results for nonlinear elliptic problems involving critical Sobolev exponents. Ann. Inst. H. PoinvaréAnalyse, Nonlin. 1, 341-350 (1984)

9. Cerami, G., Solimini, S., Struwe, M.: Some existence results for superlinear elliptic boundary value problems involving critical exponents. J. Funct. Anal. 69, 289-306 (1986)

10. Clapp, M., Weth, T.: Multiple solutions for the Brezis-Nirenberg problem. Adv. Differ. Equ. 10, 463-480 (2005)

11. Cомте, M.: Solutions of elliptic equations with critical Sobolev exponent in dimension three. Nonlinear Anal. 17, 445-455 (1991)

12. Conti, M., Merizzi, L., Terracini, S.: Remarks on variational methods and lowerupper solutions. Nonlinear Differ. Equ. Appl. 6, 371-393 (1999)

13. Conti, M., Merizzi, L., Terracini, S.: On the existence of many solutions for a class of superlinear elliptic systems. J. Differ. Equ. 167, 357-387 (2000)

14. Devillanova, G., Solimini, S.: Concentration estimates and multiple solutions to elliptic problems at critical growth. Adv. Differ. Equ. 7, 1257-1280 (2002)

15. Fortunato, D., Jannelli, E.: Infinitely many solutions for some nonlinear elliptic problems in symmetrical domains. Proc. Roy. Soc. Edinburgh. 105A, 205-213 (1987)

16. LaZer, A.C., Solimini, S.: Nontrivial solutions of operator equations and Morse indices of critical points of min-max type. Nonlinear Anal. TMA. 12, 761-775 (1988) 
17. LI, S., WANG, Z.Q.: Ljusternik-Schnirelman theory in partially ordered Hilbert spaces. Tran. Am. Math. Soc. 354, 3207-3227 (2002)

18. Marino, A., Prodi, G.: Metodi perturbativi nella teoria di Morse. Boll. U. M. I. 11(4), 1-32 (1975)

19. Rabinowitz, R.: Minimax Methods in Critical Point Theory with Applications to Differential Equations. CBMS Reg. Conf. 65. Amer. Math. Soc., Providence, RI, 1986

20. Ramos, M., SANChez, L.: Homotopical linking and Morse index estimates in min-max theorems. Manuscr. Math. 87, 269-284 (1995)

21. Ramos, M., Tavares, H., Zou, W.: A Bahri-Lions theorem revisited. Adv. Math. 222, 2173-2195 (2009)

22. Schecher, M., Zou, W.: Sign-changing critical points of linking type theorems. Trans. Am. Math. Soc. 358, 5293-5318 (2006)

23. Solimini, S.: A note on compactness-type properties with respect to Lorenz norms of bounded subsets of a Sobolev spaces. Ann. Inst. H. Poinvaré-Analyse, Nonlin. 12, 319-337 (1995)

24. Solimini, S.: Morse index estimates in minimax theorems. Manuscr. Math. 63, 421-453 (1989)

25. Viterbo, C.: Indice de Morse des poins critiques obtenus par minimax. Ann. Inst. $H$. Poinvaré-Analyse, Nonlin. 12, 221-225 (1988)

26. Zou, W.: Sign-Changing Critical Points Theory. Springer, New York, 2008

\author{
Department of Mathematics, \\ University of California, \\ Irvine, CA 92697-3875, \\ USA. \\ e-mail: mschecht@math.uci.edu \\ and \\ Department of Mathematical Sciences, \\ Tsinghua University, \\ 100084 Beijing, China. \\ e-mail: wzou@math.tsinghua.edu.cn
}

(Received November 18, 2008 / Accepted October 11, 2009)

Published online January 16, 2010 - (C) The Author(s) (2010)

This article is published with open access at Springerlink.com 